FORMATION Formation emploi

Revue française de sciences sociales

135 | Juillet-Septembre 2016

L'économie verte : rupture ou adaptation de la

formation et de l'emploi ?

\title{
Pour une anticipation stratégique des besoins en « compétences vertes » : l'outil CIPEGE
}

Towards a strategic anticipation of "green competencies": The CIPEGE tool Strategische Ermittlung des Bedarfs an „ökologischen Kompetenzen" : das

Instrument „Cipege“

Para una anticipación estratégica de las necesidades en "competencias verdes": el instrumento Cipege

Christelle L. Garrouste et Estelle Courtial

\section{(2) OpenEdition}

Journals

Édition électronique

URL : http://journals.openedition.org/formationemploi/4836

DOI : $10.4000 /$ formationemploi.4836

ISSN : 2107-0946

Éditeur

La Documentation française

Édition imprimée

Date de publication : 12 octobre 2016

Pagination : 155-177

ISSN : 0759-6340

Référence électronique

Christelle L. Garrouste et Estelle Courtial, « Pour une anticipation stratégique des besoins en " compétences vertes » : I'outil CIPEGE », Formation emploi [En ligne], 135 | Juillet-Septembre 2016, mis en ligne le 12 octobre 2018, consulté le 30 octobre 2020. URL : http://journals.openedition.org/ formationemploi/4836 ; DOI : https://doi.org/10.4000/formationemploi.4836 


\title{
Pour une anticipation stratégique des besoins en « compétences vertes » : I'outil CIPEGE
}

\begin{abstract}
Christelle L. Garrouste Enseignante-chercheure en économie au Laboratoire interdisciplinaire d'étude du politique Hannah Arendt (LIPHA, EA7373), à I'université Paris XII (UPEC université Paris-EST Créteil) - Faculté AEl (Administration et échanges internationaux) et chercheure associée au Laboratoire d'économie d'Orléans (LEO, CNRS UMR7322)

Estelle Courtial Enseignante-chercheure en automatique au Laboratoire pluridisciplinaire de recherche en ingénierie des systèmes, mécanique, énergétique (PRISME, EA 4229) de l'université d'Orléans
\end{abstract}

Résumé

Pour une anticipation stratégique des besoins en « compétences vertes » : l'outil CIPEGE

Le Centre international de prospective de l'emploi en géosciences et environnement (CIPEGE) développe, depuis 2013, un outil innovant d'anticipation, notamment, sur les tendances du marché. Il s'agit d'anticiper les stratégies en matière de besoins en compétences dans le domaine des géosciences et de l'environnement, en France. On présente ici les résultats préliminaires des premières simulations à l'horizon 2020, qui s'appuient sur une combinaison d'anticipations économétriques et d'ingénierie des systèmes.

Mots clés : écologie, gestion prévisionnelle, métier de l'environnement, employabilité, insertion professionnelle, modélisation

Abstract

Towards a strategic anticipation of "green competencies": The CIPEGE tool

The International Center for the Anticipation of Employment in Earth and Environmental Sciences (CIPEGE - Centre international de prospective de l'emploi en géosciences et environnement) is developing an anticipation tool aiming at mapping market trends and at anticipating the needs for competences in Earth Sciences and Environmental Sciences in France. This paper presents preliminary results at the horizon 2020 of this pluridisciplinary tool that combines forecasting techniques from econometrics and process control. 
Keywords: ecology, forward-looking of job management, occupation in the environmental sector, employability, transition from school to work, modelling

Journal of Economic Literature: I 20, J 24, J 62, Q 01

Traduction : Auteures.

Depuis le Sommet de la Terre de Rio, en 1992, les notions "d'économie verte " et "d'emplois verts » ont progressivement envahi les discours médiatiques et politiques. Le Programme des Nations Unies pour l'Environnement (2011) définit l'économie verte comme une économie qui engendre une amélioration du bien-être humain et de la justice sociale, tout en réduisant sensiblement les risques environnementaux et les pénuries écologiques.

Plusieurs mesures ont été adoptées afin de favoriser la transition vers une économie verte au niveau international, telles que les quotas d'émissions de gaz à effet de serre adoptés en 1995, suite à la Convention Cadre des Nations Unies sur les Changements Climatiques, le protocole de Kyoto, ou les objectifs " 20-20-20 " ${ }^{1}$ fixés par la Commission européenne en 2010. Parallèlement, les Etats se sont fixés des objectifs afin d'accélérer le "verdissement » de leur économie (comme le "Climate Change Act» de 2008 en Grande-Bretagne, ou le Grenelle de l'environnement, depuis 2007, en France). Dans le cas du Grenelle de l'environnement, des objectifs précis ont été fixés pour des domaines d'action prioritaires incluant l'amélioration énergétique des bâtiments, les transports, la réduction des consommations d'énergie et du contenu en carbone de la production, la préservation de la biodiversité, la maîtrise des risques, la préservation de la santé et le traitement des déchets.

La transition énergétique en résultant engendre de nombreux changements dans l'économie au niveau de la structure et de la dynamique des marchés, des comportements sociétaux et en matière de recherche et de formation. En outre, l'intégration des objectifs environnementaux dans l'activité économique induit un verdissement des emplois en lien avec les objectifs mentionnés précédemment (Organisation internationale du travail, 2012). Ainsi, l'économie verte inclut des emplois dont la finalité est la protection de l'environnement, des emplois qui exercent une pression faible sur l'environnement, mais aussi ceux qui exercent une pression forte tout en fournissant des secteurs verts (ex : la production d'acier dans la construction d'éoliennes) (INSEE, 2012).

Selon cette définition, les secteurs d'activité concernés par l'économie verte sont ceux utilisés pour produire des biens ou services à faible impact sur l'environnement et/ou dont la finalité est la protection de l'environnement. Néanmoins, une activité dont la finalité

1. La réduction de $20 \%$ des émissions de gaz à effet de serre de l'Union européenne par rapport à 1990, $20 \%$ d'énergies renouvelables dans la consommation d'énergie totale, et la réduction de $20 \%$ de la consommation énergétique par rapport à l'augmentation tendancielle. 
est environnementale peut avoir un effet induit négatif sur l'environnement (ex : chimie des nouveaux engrais). Réciproquement, une activité dont la finalité première n'est pas la protection de l'environnement peut exercer une pression très faible sur l'environnement du point de vue du prélèvement de matières premières, de la production de déchets, ou de rejets dans l'eau ou l'air (ex : enseignement, secteur tertiaire). Comme le montre l'INSEE (2012) dans un dossier spécial sur l'économie verte, les industries lourdes, minières et la production d'électricité n'entrent pas dans l'économie verte puisqu'elles exercent une pression très forte sur l'environnement et que leur finalité n'est pas la protection de l'environnement. À l'inverse, les activités de R\&D (recherche et développement) en efficacité énergétique sont doublement "vertes ", leur finalité étant la protection de l'environnement et leur pression sur l'environnement étant faible. En outre, l'appartenance d'un secteur à l'économie verte n'est pas figée. Notamment, en fonction de l'évolution technologique, un secteur peut voir son emprise environnementale décroître et entrer par conséquent dans l'économie verte.

Au vu de ces définitions théoriques et statistiques, les géosciences et autres sciences de l'environnement sont parmi les disciplines de formation et de recherche les plus directement affectées par la transition vers une économie verte. Les géosciences incluent l'étude de l'atmosphère, de l'hydrosphère, des océans, de la biosphère et de la Terre solide. C'est dans ce contexte que le Centre international de prospective de l'emploi en géosciences et environnement (CIPEGE) ${ }^{2}$ vise le développement d'un outil d'anticipation afin d'améliorer l'adéquation entre l'offre et la demande de compétences en géosciences et environnement.

Ici, le terme géosciences désignera à la fois les géosciences et les sciences de l'environnement. Ainsi, mesurer l'adéquation entre l'offre et la demande de compétences revient à estimer l' "employabilité " des jeunes diplômés en géosciences ${ }^{3}$. Proposer une mesure de l'employabilité n'est pas trivial en raison du caractère controversé du concept (Gazier, 1998 ; McQuaid, 2005 ; Arjona et al., 2010). Jusqu'à présent, aucune mesure n'est parvenue à en refléter toutes les dimensions. Au contraire, McQuaid (2005) précise que l'on peut regrouper en deux catégories les types de mesures de l'employabilité en fonction de la perspective adoptée. La première se focalise sur les caractéristiques individuelles et les compétences et se réfère à l'aptitude d'un individu à trouver un travail. La seconde prend en compte des facteurs exogènes (comme les institutions du marché du travail, le statut socio-économique de l'individu, etc.) qui peuvent influencer la probabilité qu'un indi-

2. Le CIPEGE a été défini comme une action du LABEX (laboratoire d'excellence) Voltaire (ANR-10LABS-100-01) dédiée à l'étude des fluides géologiques et environnementaux et aux interactions entre Terre et atmosphère. Alors que les finalités économiques du LABEX concernent les ressources géologiques et l'environnement (sols, eau, aquifères, agro-écosystèmes, qualité de l'air), le CIPEGE a pour ambition de jeter les ponts entre le monde académique et les industries pour parvenir à une meilleure adéquation entre formation et emploi.

3. L'approche adoptée d'identification des besoins en compétences s'inscrit dans les travaux relatifs au capital humain et diffère d'autres approches, comme la GPEC (gestion prévisionnelle des emplois et des compétences) en ressources humaines, par exemple. 
vidu a de trouver un emploi, d'évoluer entre deux emplois et de progresser dans son travail. Selon De Grip (2004), ces facteurs sont des conditions pour qu'un travailleur puisse "effectuer» son employabilité ("effectuation conditions»). La littérature prend également en compte le rôle joué par la durée de transition entre le moment de l'obtention du dernier diplôme et le premier emploi (Boateng et al., 2011), le degré d'adéquation entre la formation et le poste occupé ou le type de contrat (plein temps ou temps partiel; contrat à durée indéterminée ou à durée déterminée) (Arjona et al., 2010).

L'employabilité est donc la capacité d'obtenir un premier emploi, de maintenir un emploi et d'obtenir un nouvel emploi (Cedefop, 2008). En d'autres termes, l'employabilité d'un jeune diplômé est sa prédisposition à exhiber les attributs qu'un employeur anticipe comme essentiels pour le bon fonctionnement de son organisation (Harvey, 1998). L'employabilité est aussi définie comme une combinaison de capacité et de volonté à être et à demeurer attractif pour le marché du travail (De Grip, 2004). Pour un individu donné, son employabilité va dépendre de ses connaissances, de ses compétences, de son attitude, et de la manière dont il en fait usage et les présente à un futur employeur (Hillage, 1998).

Dans le cadre de l'outil CIPEGE, nous retenons une approche de l'employabilité par type de poste, en définissant l'employabilité comme la capacité des jeunes diplômés des universités françaises en géosciences à être employés à un poste qui exploite leurs compétences en géosciences, tout en tenant compte des tendances de la demande internationale pour ce type de compétences ${ }^{4}$. En effet, de nombreux travaux empiriques démontrent que les retours positifs de l'investissement en éducation (en termes de salaire) sont encore plus forts lorsqu'il existe une adéquation entre les compétences acquises durant la formation et le poste occupé pour les diplômés à partir du niveau Bac + 2 (par exemple, pour la France, Couppié, Giret et Lopez, 2009, p. 54).

Cet article présente les premiers résultats de l'outil d'anticipation développé par le CIPEGE. Alors que la deuxième partie sera dédiée à un aperçu de l'emploi dans le domaine des géosciences et environnement en Europe et en France, la troisième partie présentera la méthodologie sous-jacente à l'outil CIPEGE. Cet outil d'anticipation combine des stratégies de modélisation utilisées traditionnellement en économie (théorie d'appariement et multinomial logit) et des stratégies de commande utilisées en ingénierie des systèmes (modèle interne et commande prédictive). Les résultats de nos

4. Ce faisant, cette conception de l'employabilité s’inscrit dans le prolongement de travaux menés par la Commission européenne pour mesurer la capacité des universités européennes à délivrer les compétences exigées par le marché du travail (cf. Benchmark Employabilité des jeunes diplômés à l'horizon 2020, (Bruxelles, 10 et 11 mai 2012 : http://www.consilium.europa.eu/uedocs/cms_data/docs/pressdata/en/educ/130142. pdf. Cette définition privilégie la capacité à faire valoir sa formation à travers son emploi et permet de mener des évaluations ciblées de l'efficacité des politiques publiques. Elle ne s'oppose pas à des définitions alternatives de l'employabilité, mais celles-ci sont souvent plus difficilement quantifiables, notamment en termes d'analyse d'impact. 
simulations seront présentés et interprétés dans la quatrième partie. La dernière section servira enfin à discuter les potentialités et les limites de l'outil CIPEGE au vu des résultats obtenus.

\section{Les géosciences et l'emploi : secteurs porteurs et secteurs en berne}

Après une vue d'ensemble introductive des similitudes et divergences des marchés français et européens en termes de secteurs porteurs ou en berne, pour les diplômés en géosciences, nous montrerons que les diplômés de Master ont de réelles opportunités, en France, dans un contexte de reprise économique.

\subsection{La transition énergétique et l'emploi : quelles spécificités françaises au regard de nos partenaires européens ?}

À l'aide de son modèle macroéconomique E3ME (Economics, Environment and Energy Model for Europe) (cf. Annexe 1 en version électronique), Cambridge Econometrics (2011) a procédé à une analyse approfondie des conséquences sur l'emploi de la mise en œuvre des politiques européennes pour atteindre les objectifs environnementaux "20-20-20 ». Les résultats révèlent des tendances très hétérogènes par secteur d'activité. Alors que certains secteurs, comme le fer, l'acier, le ciment et le pétrole, connaîtront une baisse de l'emploi d'ici 2020, d'autres, comme les énergies renouvelables, la construction et le transport, enregistreront une croissance de l'emploi.

Les professions qui bénéficieront le plus d'une transition vers une plus faible émission de carbone sont l'ingénierie et les professions techniques liées à la production d'électricité à partir de ressources naturelles (vent, eau, soleil), soit des professions hautement qualifiées (Wilson, 2010). Le rapport souligne également le fait que la demande devrait s'accroître pour certains types d'ingénieurs et de techniciens, qui, outre leur haute qualification dans leur discipline (ex : ingénierie électronique), seront capables d'appliquer leurs connaissances dans un contexte professionnel « verdissant " et à des postes aussi variés que la $\mathrm{R} \& \mathrm{D}$, le design, la maintenance et le management.

Nous avons exécuté l'algorithme du modèle E3ME afin d'obtenir la demande d'emploi par secteur pour les diplômés des universités, à la fois au niveau européen agrégé et pour la France. Les courbes de tendance à l'horizon 2020, présentées dans le Graphique 1, illustrent les similitudes et les différences entre le marché du travail en France et le marché du travail européen au niveau agrégé.

La France suit les tendances européennes dans différents secteurs clefs d'extraction et d'exploitation des ressources minières, tels que l'industrie des produits minéraux non-métalliques et l'industrie du charbon, avec une tendance à la hausse du nombre de la demande 
de travailleurs possédant un diplôme de niveau universitaire et à la baisse s'agissant des travailleurs ayant un faible niveau de diplôme. Notons toutefois que la baisse observée, en France, dans l'industrie du charbon, est bien plus prononcée qu'au niveau européen.

Pour les industries d'extraction et d'exploitation des métaux de base, du pétrole et du gaz, la France révèle une tendance inversée par rapport à la moyenne européenne. Alors que l'emploi des diplômés de l'enseignement supérieur va progressivement diminuer en Europe, dans les secteurs des métaux de base, du pétrole et du gaz, il va augmenter en France.

Au-delà de ces tendances globales (représentées par une ligne pleine sur les graphiques), il est intéressant d'observer les tendances au niveau désagrégé du type de poste, afin d'avoir une idée plus précise du niveau de diplôme qui sera demandé. Si l'on examine les industries des métaux de base, on observe que les emplois en expansion dans les années à venir sont essentiellement des emplois de scientifiques et ingénieurs associés, c'est-à-dire des diplômés de niveau $\mathrm{Bac}+3$ ou $\mathrm{Bac}+4$, au détriment des scientifiques et ingénieurs diplômés de niveau $\mathrm{Bac}+5$ ou $\mathrm{Bac}+8$. Cette tendance en faveur de compétences techniques non spécialisées confirme les résultats obtenus par Cambridge Econometrics (2011) au niveau européen. Ces résultats très généraux fournissent un aperçu du type de diplômes qui seront prisés par le marché dans les années à venir, en termes de discipline et de niveau.

\subsection{L'emploi en géosciences en France : le Master, garant d'un emploi en lien avec la formation}

Après avoir analysé les tendances de l'emploi dans les industries traditionnellement associées aux géosciences, nous avons étudié les parcours professionnels des diplômés en géosciences et environnement, afin de vérifier si leur diplôme les menait vers ces industries. Une première analyse des données individuelles annualisées de l'enquête Emploi de l'INSEE, sur la période 2003-2011, fournit un aperçu de la répartition des diplômés en sciences de la Terre et de l'univers, par industrie, pour chaque niveau de diplôme. À partir de la Licence $(\mathrm{Bac}+3)$, le secteur principal d'insertion des géoscientifiques est l'éducation $(40 \%$ des $\mathrm{Bac}+3,62 \%$ des $\mathrm{Bac}+4,21 \%$ des $\mathrm{Bac}+5$ et $30 \%$ des $\mathrm{Bac}+8)$, suivi de près par le secteur des services professionnels, ie les emplois de consulting et d'expertise. Le rôle important du secteur a émergé en 2008, suite à la crise économique, et a engendré une hausse des emplois temporaires hautement qualifiés (Cutuli, 2013 ; Oliver, 2012 ; O’Connor, 2013). Dans le cas des diplômés de niveau Bac +2 , le secteur le plus attractif est l'administration publique et la défense (27\%). Le Tableau 1 résume cette distribution.

Notons que le niveau de diplôme qui garantit le mieux un emploi dans des secteurs en relation avec la formation (en gris dans le Tableau 1) est le Master $(\mathrm{Bac}+5)$, avec des emplois dans treize secteurs dits " verts » ou " verdissants » sur les dix-neuf secteurs d'insertion effective. Arrivent ensuite, en ordre décroissant, les $\mathrm{Bac}+4, \mathrm{Bac}+8$ et $\mathrm{Bac}+2 / 3$. 
Graphique 1. Evolution de la demande de diplômés du tertiaire $(\mathrm{Bac}+2$ à $\mathrm{Bac}+8)$ entre 2000 et 2020, par secteur d'activité, dans l'ensemble de l'Union européenne (EU27) et en France

Nombres de diplômés demandés en UE
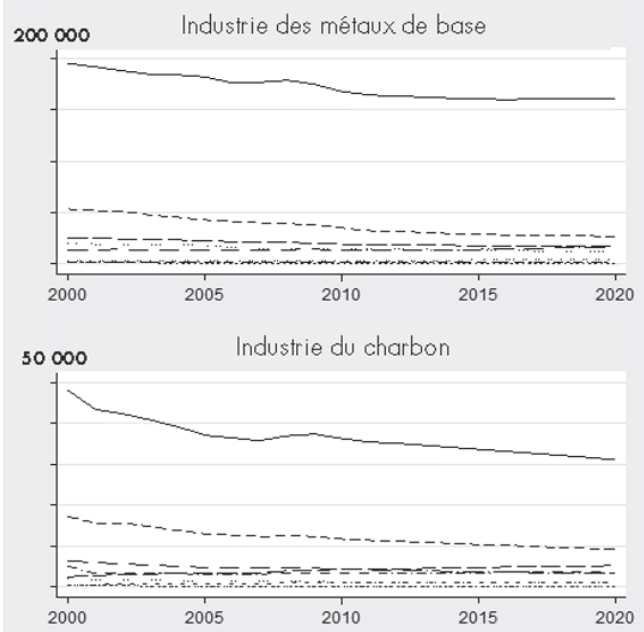

300000 Industrie des produits minéraux non-métalliques

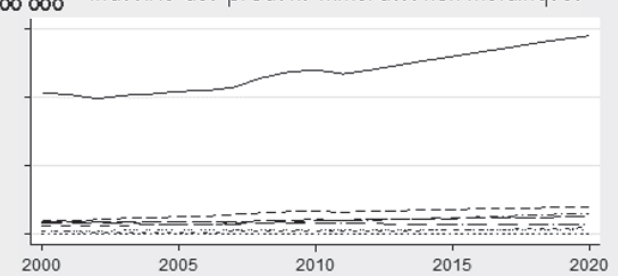

$60000 \quad$ Industrie du pétrole et du gaz

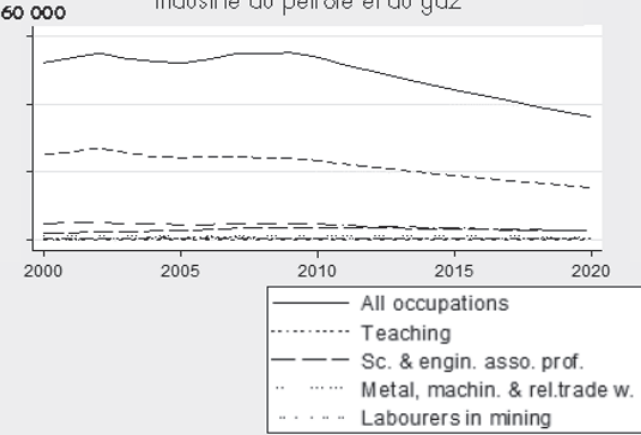

\section{Nombres de diplồmés demandés en France}
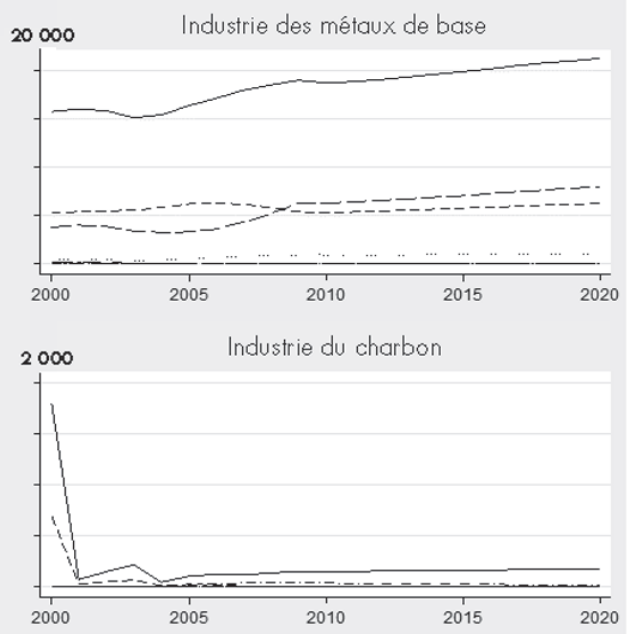

40000 Industrie des produits minéraux non-métalliques
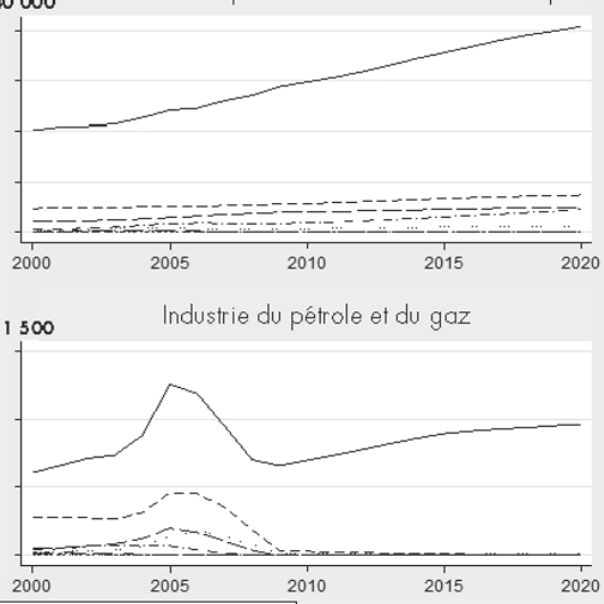

---- Sc. \& engin. prof.

$-\ldots$ Other professionals

- -.-. Extract. \& build. w.

-....... Operators 
Note : «All occupations » : Tous types d'occupations/fonctions quel que soit le niveau d'études ; «Sc. \& engin. prof. » : Scientifiques et ingénieurs professionnels; « Sc. \& engin. asso. prof. » : Scientifiques et ingénieurs associés professionnels ; « Metal. machin. \& rel. trade w. » : machinistes et autres travailleurs et commerciaux en métallurgie ; " Labourers in mining » : travailleurs miniers ; « Teaching »: Enseignement ; « Otherprofessionals » : Autres professionnels qualifiés ; "Extract. \&build. w. » : Travailleurs en extraction et construction ; «Operators » : Opérateurs. Les résultats pour les autres secteurs d'activité (industries) sont disponibles auprès des auteurs.

Les cadrans graphiques de gauche correspondent à la demande moyenne des 27 pays européens (y compris la France) pour les compétences de niveau universitaire, par secteur d'activité. Les cadrans graphiques de droite présentent la demande française pour les compétences de niveau universitaire dans les mêmes secteurs d'activité. La ligne pleine représente la demande totale par secteur, tous types d'emplois et de niveau de qualifications confondus. Cette ligne sert de référence cardinale afin de mieux percevoir la demande relative pour les diplômés de l'enseignement supérieur. Pour des raisons visuelles, seuls les types d'emploi les plus importants (en termes de nombre) sont présentés.

Source : Estimations des auteurs à partir du modèle E3ME (Energy-Environnement-Economy Model For Europe).

De plus, certains secteurs ne recrutent des géoscientifiques qu'au-delà d'un certain niveau de diplôme. C'est le cas des secteurs du bois et du papier (uniquement des Bac +4 ), des secteurs de l'énergie hydraulique, de l'industrie pétrolière et gazière $(\mathrm{Bac}+4 \mathrm{et} \mathrm{Bac}+5)$.

Si l'on considère que l'employabilité d'un diplômé ne se limite pas à sa capacité à trouver un emploi, mais consiste à trouver un emploi dans son domaine de formation, le fait qu'au moins $25 \%$ des diplômés en géosciences soient employés dans des secteurs d'insertion non directement liés à la thématique initiale de formation signale une inadéquation entre la formation offerte et la formation demandée (" mismatch" de compétences) sur ce marché. Cette perte de compétences en géosciences, au profit de secteurs non directement demandeurs de ce type de compétences, peut pénaliser les secteurs demandeurs. En outre, la non-exploitation des compétences acquises durant la formation constitue une source potentielle d'insatisfaction des travailleurs, surtout dans les premières années suivant l'obtention du diplôme (Boras, Legay, Romani, 2008). 
Tableau 1. Distribution des emplois pour les diplômés en sciences de la Terre et de l'univers, par niveau de diplôme et secteur d'activité

\begin{tabular}{|c|c|c|c|c|c|}
\hline & $\mathrm{Bac}+2$ & $\mathrm{Bac}+3$ & $\mathrm{Bac}+4$ & $\mathrm{Bac}+5$ & $\mathrm{Bac}+8$ \\
\hline Agriculture & $<0,1 \%$ & $2 \%$ & $0,2 \%$ & $4 \%$ & $0,4 \%$ \\
\hline Transport aérien & $<0,1 \%$ & $<0,1 \%$ & $<0,1 \%$ & $3 \%$ & $<0,1 \%$ \\
\hline Métaux de base & $<0,1 \%$ & $4 \%$ & $<0,1 \%$ & $2 \%$ & $<0,1 \%$ \\
\hline Produits chimiques & $5 \%$ & $<0,1 \%$ & $1 \%$ & $<0,1 \%$ & $<0,1 \%$ \\
\hline Construction & $4 \%$ & $5 \%$ & $0,2 \%$ & $5 \%$ & $4 \%$ \\
\hline Education & $9 \%$ & $40 \%$ & $62 \%$ & $21 \%$ & $30 \%$ \\
\hline Ingénierie électronique & $<0,1 \%$ & $<0,1 \%$ & $<0,1 \%$ & $1 \%$ & $3 \%$ \\
\hline Transport routier & $4 \%$ & $3 \%$ & $<0,1 \%$ & $<0,1 \%$ & $<0,1 \%$ \\
\hline Carburants fabriqués & $<0,1 \%$ & $<0,1 \%$ & $<0,1 \%$ & $<0,1 \%$ & $7 \%$ \\
\hline Ingénierie mécanique & $2 \%$ & $1 \%$ & $<0,1 \%$ & $9 \%$ & $19 \%$ \\
\hline Produits métalliques & $<0,1 \%$ & $3 \%$ & $0,2 \%$ & $<0,1 \%$ & $<0,1 \%$ \\
\hline Motorisation/véhicules & $4 \%$ & $<0,1 \%$ & $<0,1 \%$ & $1 \%$ & $<0,1 \%$ \\
\hline Pétrole et gaz & $<0,1 \%$ & $<0,1 \%$ & $1 \%$ & $1 \%$ & $<0,1 \%$ \\
\hline Services professionnels / experts & $8 \%$ & $4 \%$ & $6 \%$ & $16 \%$ & $16 \%$ \\
\hline Transport de l'eau & $<0,1 \%$ & $<0,1 \%$ & $0,4 \%$ & $2 \%$ & $<0,1 \%$ \\
\hline Bois et papier & $<0,1 \%$ & $<0,1 \%$ & $1 \%$ & $1 \%$ & $<0,1 \%$ \\
\hline Administrations publiques et défense & $27 \%$ & $4 \%$ & $7 \%$ & $11 \%$ & $11 \%$ \\
\hline Banque et finance & $1 \%$ & $<0,1 \%$ & $2 \%$ & $1 \%$ & $<0,1 \%$ \\
\hline Communications & $1 \%$ & $4 \%$ & $<0,1 \%$ & $<0,1 \%$ & $<0,1 \%$ \\
\hline Services informatiques & $4 \%$ & $<0,1 \%$ & $3 \%$ & $5 \%$ & $0,4 \%$ \\
\hline Distribution & $5 \%$ & $<0,1 \%$ & $0,2 \%$ & $6 \%$ & $<0,1 \%$ \\
\hline Santé et travail social & $9 \%$ & $7 \%$ & $4 \%$ & $<0,1 \%$ & $1 \%$ \\
\hline Hôtels et traiteurs & $<0,1 \%$ & $1 \%$ & $3 \%$ & $<0,1 \%$ & $<0,1 \%$ \\
\hline Assurance & $<0,1 \%$ & $<0,1 \%$ & $1 \%$ & $<0,1 \%$ & $3 \%$ \\
\hline Autres services & $9 \%$ & $11 \%$ & $3 \%$ & $10 \%$ & $3 \%$ \\
\hline $\begin{array}{l}\text { Services en gestion et administration } \\
\text { des affaires }\end{array}$ & $<0,1 \%$ & $<0,1 \%$ & $1 \%$ & $0,3 \%$ & $3 \%$ \\
\hline Produits pharmaceutiques & $7 \%$ & $4 \%$ & $<0,1 \%$ & $2 \%$ & $0,4 \%$ \\
\hline Imprimerie et publication & $<0,1 \%$ & $4 \%$ & $<0,1 \%$ & $<0,1 \%$ & $<0,1 \%$ \\
\hline Commerce de detail & $1 \%$ & $6 \%$ & $3 \%$ & $<0,1 \%$ & $<0,1 \%$ \\
\hline $\begin{array}{l}\text { Nombre d'observations annuelles } \\
\text { moyennes (1990-2011) }\end{array}$ & 2010 & 2402 & 6756 & 4514 & 3378 \\
\hline
\end{tabular}

Note : Les secteurs d'activité en gris correspondent aux secteurs traditionnellement associés aux formations en sciences de la Terre et de l'univers (liste établie en concertation avec le ministère de l'Education supérieure et de la Recherche et l'Observatoire des sciences de l'univers d'Orléans).

Source : Estimation des auteurs à partir des données individuelles de l'enquête Emploi de l'INSEE sur la période 1990-2011. 


\section{Le CIPEGE : vers un outil d'anticipation interdisciplinaire de l'employabilité}

L'outil CIPEGE est composé de deux modèles consistant respectivement à estimer l'employabilité, en termes de capacité d'appariement de l'offre et de la demande de compétences (sous-partie 2.1), et à estimer la capacité des universités à adapter le flux de leurs diplômés à cette employabilité de référence, en tenant compte des choix individuels de parcours des étudiants (modèle logit multinomial). Les deux modèles économétriques sont reliés par un processus d'optimisation de l'offre de compétences en fonction d'une trajectoire de référence (ici, l'employabilité de référence), selon une approche de commande prédictive (sous-partie 2.2).

\subsection{Modéliser l'employabilité}

L'objectif de l'outil CIPEGE étant d'anticiper l'employabilité des jeunes diplômés des universités françaises en géosciences et sciences de l'environnement, il est dans un premier temps nécessaire de définir le modèle retenu pour capturer cette employabilité (cf. Encadré 1).

\section{Encadré 1 : Modèle de l'employabilité de référence}

Soit $y_{j}^{F}$ le nombre d'individus employés en France qui ont un diplôme en géosciences de niveau $\mathrm{Bac}+j$, avec $j \in\{2,3,4,5,8\}$. Un niveau $j=2$ correspond à un Diplôme d'Etudes Universitaires Générales (DEUG) ou Diplôme Universitaire de Technologie (DUT), $j=3$ à une Licence, $j=4$ à une Maîtrise (Master I depuis 2004), $j=5$ à un Diplôme d'Etudes Approfondies (DEA), à un Diplôme d'Etudes Supérieures Spécialisées (DESS) ou Master II (depuis 2004) et $j=8$ à un Doctorat. Pour chaque niveau de diplôme $j$, le nombre de diplômés employés en France à l'instant $t$ est :

$y_{j}^{F}(t)=S_{j}^{F}(t)-u n_{j}^{F}(t)(1)$

avec $S_{j}^{F}$ le stock de diplômés en géosciences parmi la population active et $u n_{j}^{F}$ le stock de diplômés en géosciences au chômage sur le marché français.

L'employabilité est déterminée par le nombre de diplômés en géosciences employés, en France, à un poste exploitant leurs compétences dans ce domaine, en tenant compte de l'évolution du nombre de demandeurs d'emplois avec un diplôme en géosciences et du nombre de postes vacants dans ce domaine au niveau européen. La dynamique de l'emploi est formalisée par l'équation (2) : 


$$
y_{j}^{F}(t+1)=m_{j}^{E U}(t)+(1-\delta) y_{j}^{F}(t)=\mu\left(u n_{j}(t)\right)^{a}\left(v_{j}(t)\right)^{b}+(1-\delta) y_{j}^{F}(t)
$$

avec $y_{j}^{F}(t+1)$ le nombre d'employés en France parmi les diplômés en géosciences à l'instant $t+1, m_{j}^{E U}(t)$ le paramètre de " matching » (appariement) à l'instant $t$ sur le marché européen, $u n_{j}$ le stock de chômeurs parmi les diplômés en géosciences en Europe ; $v_{i}$ le nombre de postes vacants dans le domaine des géosciences au niveau $B a c+j$, pour tout $i \in\{2,3,4,5,8\}$ et $\delta$ le flux de destruction d'emplois. Le terme d'adéquation sur le marché du travail européen, $m_{j}^{E U}(t)$, est dérivé d'une fonction de « matching », telle que définie par Mortensen et Pissarides (1994), c'est-à-dire une fonction Cobb-Douglas croissante, concave et homogène de degré 1 $(a+b \approx 1)$, dont les paramètres $\mu$, a et $b$ sont constants et positifs. L'évolution du chômage est donnée par:

$$
u \operatorname{un}_{j}=\delta\left(1-u n_{j}\right)-m_{j}^{E U}\left(u n_{j}, v_{j}\right) \text {. (3) }
$$

Supposons qu'il existe une technologie d'appariement, c'est-à-dire une description de la relation entre les entrées, la recherche et l'activité de recrutement, et la sortie du processus d'appariement, le débit avec lequel chômeurs et emplois vacants forment un nouveau singleton emploi-travailleur (Pissarides et Mortensen, 1999). Sous I'hypothèse que cette technologie d'appariement présente des rendements constants, cette équation a une solution stable unique pour tous les taux de vacance $v$ :

$u n_{j}=\frac{\delta}{\left[\delta+m_{j}^{E U}\left({ }^{v_{j}} / u n_{j^{\prime}} 1\right)\right]}=\frac{\delta}{(\delta+\lambda(\theta))}(4)$

où $\theta=\left(v_{j} / u n_{j}\right)$ dénote les tensions sur le marché et $\lambda(\theta)=m_{j}^{E U}\left(v_{j} / u n_{j}, 1\right)$ le risque d'être au chômage. Représenter l'équation (4) dans un espace vacance-chômage génère une courbe de Beveridge, c'est-à-dire une relation négative entre les postes vacants et le chômage par propriété de la fonction de « matching » (voir les détails dans Pissarides et Mortensen, 1999).

Les données montrent que le flux de destruction d'emplois, $\delta$, n'est pas constant, en particulier sur la fréquence des cycles d'affaires (Davis, 1996). Selon Pissarides et Mortensen (1999), cela permet de faire varier la productivité future de l'emploi $\rho$ en fonction de la valeur relative (en termes de compétences requises) $x$ du produit ou du service; parce que $x \in[0,1], \rho x$, peut prendre plus de deux valeurs. Selon Pissarides et Mortensen (1999), Mortensen et Pissarides (1994) et Cole (1996), le fait de considérer $\rho$ (la productivité future) comme un processus stochastique caractérisant un choc global, est compatible avec le temps de destruction et de création d'emplois signalé par les séries sur l'emploi (Davis, 1996).

Quand un chômeur et un employeur avec une vacance se rencontrent, les négociations salariales commencent. Il en résulte un salaire $w(x)$ qui divise les quasi-rentes associées à un appariement entre le travailleur et l'employeur, en fonction de la valeur de $x$. La valeur d'un emploi occupé est une fonction de la productivité du travail future, du salaire négocié et de la probabilité de destruction de l'emploi. La création d'emplois a lieu si tous les loyers de la création de nouveaux postes vacants sont épuisés, c'est-à-dire si $v_{j}=0$. Un emploi est détruit si sa productivité idiosyncrasique tombe en dessous d'un niveau critique $r s$ (productivité de réservation d'équilibre), c'est-à-dire si $x<r s$. 
Tous les paramètres du modèle sont estimés à l'aide des données individuelles anonymes de l'enquête Emploi de l'INSEE (1990-2011), à l'exception du paramètre de " matching $\left(m_{\dot{f}}^{E U}\right)$, estimé à l'aide de la version étendue du modèle E3ME développé par Cambridge Econometrics (1974-2012). Le modèle E3ME est un modèle à correction d'erreur, qui intègre l'erreur d'estimation à chaque instant $t$ dans l'estimation des coefficients en $t+1$. Les données de l'INSEE nous permettent d'estimer directement le flux de destruction d'emplois, $\delta$, ainsi que l'emploi actuel des diplômés en géosciences en France, pour chaque niveau de diplôme $B a c+j, y_{j}^{F}(t)$, par la méthode des moindres carrés ordinaires, en contrôlant chaque secteur d'activité.

L'utilisation du E3ME pour l'estimation du paramètre de matching (appariement) nous permet de capturer, de manière indirecte, l'impact des chocs environnementaux et énergétiques sur la demande de compétences en géosciences (cf. Annexe 2 en version électronique). Nous utilisons le E3ME au niveau agrégé (c'est-à-dire pour l'ensemble des pays européens) afin de générer le stock de demande pour les travailleurs les plus hautement qualifiés (i.e. CITE5-6), par secteur (selon la classification NACE - Nomenclature statistique des activités économiques) et par niveau d'occupation (selon la classification CITP-08 - Classification internationale type des professions). À l'aide des données individuelles de l'enquête Emploi de l'INSEE, de 1990 à 2011, nous identifions les secteurs d'exercice et les niveaux d'occupation des diplômés en géosciences, en France, selon leur niveau de diplôme (cf. Tableau 1). Le nombre de travailleurs demandés en géosciences, en Europe, avec un niveau de compétences $j$, $m_{j}^{E U}$, est obtenu en fusionnant les données précédemment estimées à l'aide du modèle E3ME, à celles de l'INSEE, sur la base de ces matrices activité/occupation, par niveau de diplôme. Le paramètre $m_{j}^{E U}$ est ensuite intégré à l'équation (2) afin d'estimer le nombre de diplômés en géosciences, au niveau $B a c+j$, qui seront employés à la période suivante.

Cette valeur anticipée de la demande des marchés pour des diplômés en géosciences constitue notre employabilité de référence, c'est-à-dire l'objectif à atteindre (voir, pour les diplômés de Master, le Graphique 2). Il s'agit principalement de comprendre comment le système universitaire français peut ajuster son offre de diplômés en géosciences afin de répondre à la demande des marchés. Il est donc nécessaire de développer un outil d'anticipation de la demande qui va être sensible à des ajustements de l'offre de diplômés en géosciences. C'est ce que l'outil CIPEGE se propose de faire à l'aide d'une approche de commande prédictive (Model Predictive Control). 


\section{Graphique 2. Employabilité des diplômés de Master $(\mathrm{Bac}+5)$ en géosciences} de 2003 à 2025

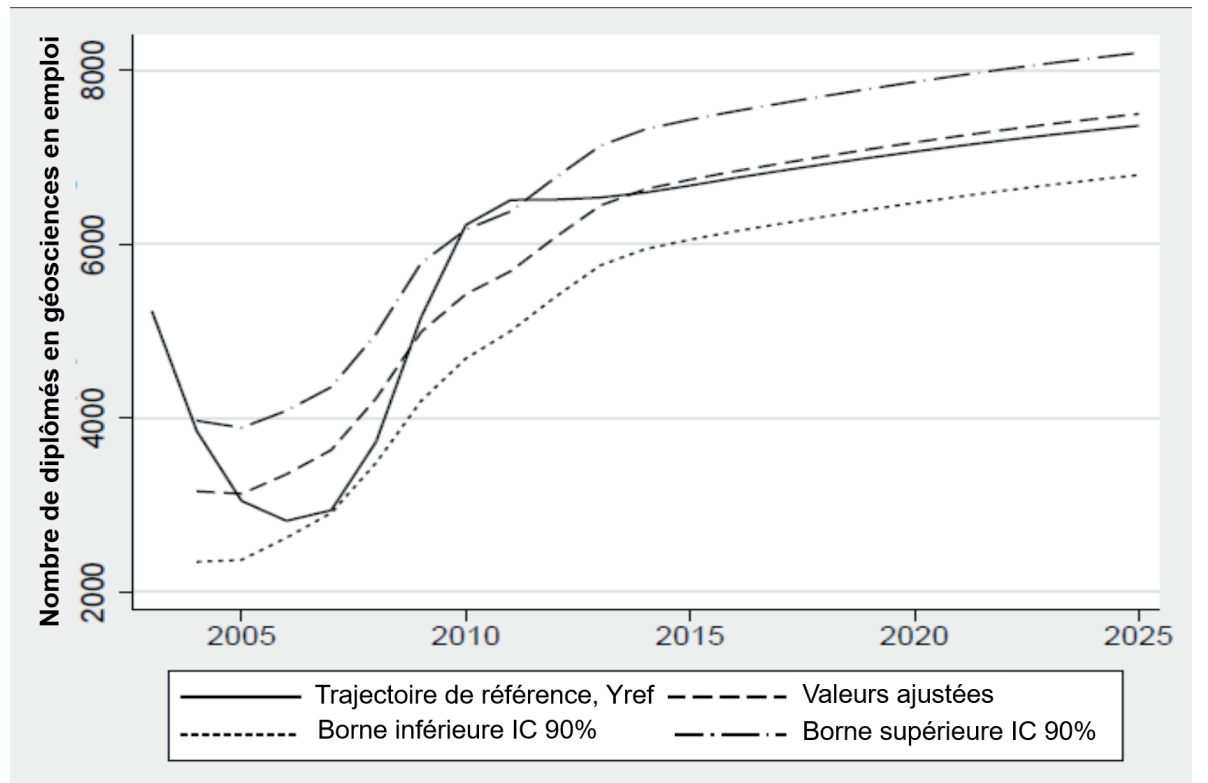

Note : La ligne pleine représente l'employabilité d'un diplômé en géosciences d'une université française, en tenant compte de la demande pour les compétences en géosciences au niveau européen. Cette courbe correspond à la trajectoire de référence que les universités françaises doivent avoir à l'esprit lorsqu'elles mettent en place des mesures visant la formation en géosciences. Les valeurs jusqu'en 2012 sont estimées à partir de données observées. Au-delà de 2012, les valeurs sont prédites sur la base d'une extrapolation linéaire dont les valeurs prédites sont représentées par la ligne pointillée. Cette extrapolation est bornée dans un intervalle de confiance (IC) de 90 \% (cf. bornes inférieure et supérieure).

Source : Estimation des auteurs du modèle dynamique de l'emploi (cf. équation 2, encadré 1) à partir des données estimées avec le E3ME de 1974 à 2012, et des enquêtes Emploi annuelles de l'INSEE de 2003 à 2012.

\subsection{Anticiper l'évolution de l'employabilité : approche de commande prédictive}

Dans le domaine de l'ingéniérie des procédés, les commandes prédictives font partie des approches de commandes avancées des procédés les plus utilisées (Alessio, 2009; Qin, 2003). Initialement développées pour des procédés linéaires dans les années 1970, dans le milieu pétrochimique, les commandes prédictives (CP) se sont progressivement imposées dans de nombreux domaines industriels (automobile, agroalimentaire, énergie - nombreuses applications pour la gestion optimisée de la consommation énergétique des bâtiments). 
Toutes les CP ont en commun les trois points suivant : une trajectoire de référence (ici, l'employabilité des diplômés), un modèle du procédé (ici, le modèle mathématique estimant le nombre d'étudiants à former pour répondre à la demande du marché) et un critère d'optimisation (ici, l'ajustement du nombre d'inscrits en début de parcours afin d'atteindre le nombre de diplômés désiré). La stratégie des CP se base sur le principe de l'horizon glissant ("moving or receding horizon") et l'objectif de commande est formulé en un problème d'optimisation non-linéaire (Camacho, 2007). À l'instant courant $t$, la sortie du procédé est mesurée. L'utilisation explicite d'un modèle du procédé permet de prédire le comportement du procédé sur un horizon fini, $N_{p}$, appelé horizon de prédiction. Il s'agit alors de déterminer une séquence de $N_{c}$ commandes, où $N_{c}$ est l'horizon de commande, permettant de minimiser la différence entre la trajectoire de référence et la sortie prédite par le modèle sur l'horizon $N_{p}$. Seule la première composante de cette séquence est réellement appliquée au procédé. À l'instant suivant $t+1$, l'horizon de prédiction glisse d'une période d'échantillonnage, les mesures sont actualisées et toute la procédure est répétée. Le terme "prédiction » est ici synonyme d'anticipation ou prévision. Etant donnée sa formulation en un problème d'optimisation, la CP peut facilement prendre en compte des contraintes, notamment sur les états et les entrées (commandes) du procédé.

La trajectoire de référence, $y_{\text {ref }}$ (cf. Encadré 1), correspond au comportement souhaité du procédé. Dans notre cas, $y_{\text {ref }}$ correspond à l'employabilité des diplômés des universités françaises en géosciences et environnement. Le "procédé " est la capacité des universités françaises à produire les compétences en géosciences et environnement requises par le marché (aussi bien en qualité -niveau et type de formation - qu'en quantité - nombre de diplômés). Cette capacité équivaut au nombre de diplômés en géosciences formés par les universités françaises chaque année, c'est-à-dire à chaque instant $t$. L'objectif de commande est d'améliorer l'employabilité des diplômés en géosciences et environnement en agissant sur le procédé, par l'ajustement du nombre de diplômés entrant sur le marché du travail chaque année.

Les prédictions basées sur des données sont inévitablement sujettes à des perturbations et à des erreurs de modélisation. Afin de gagner en robustesse, nous utilisons une structure de commande à modèle interne (Internal Model Control), largement reconnue en Automatique, que nous avons adaptée à notre problématique d'employabilité (cf. Graphique 3). 


\section{Graphique 3. Structure de commande CIPEGE}

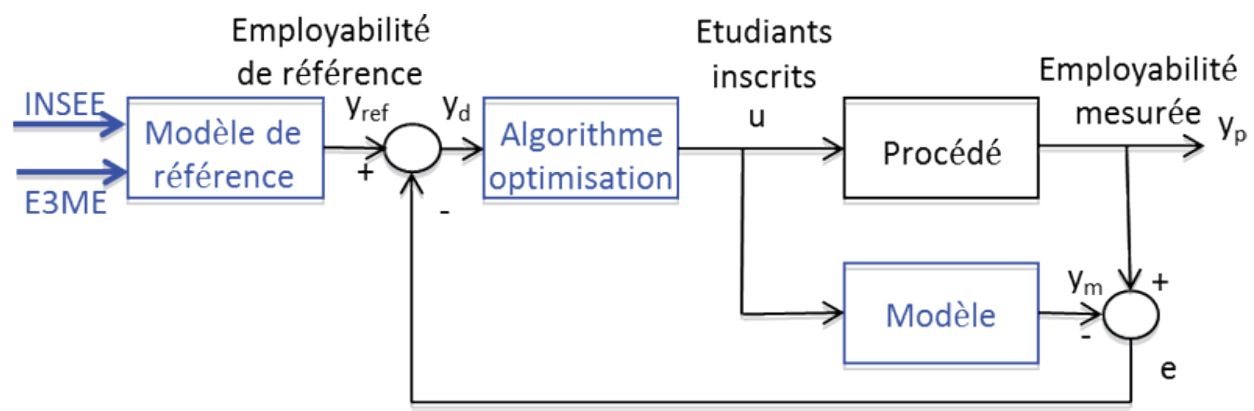

Cipege : Centre international de prospective de l'emploi en géosciences et environnement .

Source : Auteures.

Le modèle (aussi appelé modèle interne) décrit mathématiquement le comportement du procédé. Afin de prédire l'évolution du nombre de diplômés en géosciences que la France va pouvoir produire sur un horizon $N_{p}$, le modèle interne que nous avons choisi est un modèle logit multinomial/conditionnel (McFadden, 1974).

Supposons qu'avant chaque rentrée universitaire, les étudiants disposent d'un choix discret $Y_{i}$ entre $A$ options de parcours, dont une option de sortie, une option de redoublement et une option de poursuite. Soit $U_{i a}$ la fonction d'utilité de l'étudiant $i$ choisissant l'option $a$, qui dépend d'une composante systématique $\eta_{i a}$ et d'une composante aléatoire $\varepsilon_{i a}$, telle que le vecteur $\varepsilon_{i}=\left(\varepsilon_{i 1}, \ldots, \varepsilon_{i A}\right)$ a une distribution normale multivariée avec un vecteur de moyennes nulles et une matrice de corrélations arbitraires $R$. Nous supposons que les étudiants sont rationnels, c'est-à-dire qu'ils maximisent leur utilité. L'étudiant $i$ choisira l'alternative $a$ si $U_{i a}$ est la plus grande parmi $\left\{U_{i 1}, \ldots, U_{i A}\right\}$. La probabilité que l'individu $i$ choisisse l'alternative $a$ est :

$\pi_{i a}=\operatorname{Pr}\left[Y_{i}=a\right]=\operatorname{Pr}\left[\max \left(U_{i 1}, \ldots, U_{i A}\right)=U_{i a}\right]$

Ce modèle présente l'avantage de permettre de corréler les utilités qu'un étudiant assigne aux différentes options de parcours.

Le nombre d'étudiants $w_{s}$ inscrits chaque année en filière $s$, va dépendre de cette probabilité de parcours individuel et du flux d'étudiants observé. Ainsi, le nombre d'étudiants choisissant l'option $a$ chaque année est donné par :

$\hat{N}(t)=\sum_{s=1}^{S} w_{s} P_{s a}=y(t)_{m}$

où $P_{s a}$ est la probabilité (résultat de l'équation (5)) qu'un étudiant en filière $s$ choisisse l'alternative $a$.

L'hypothèse de rationalité inhérente à ce modèle est difficilement vérifiable, mais indispensable pour la construction de notre analyse. L'un des objectifs de l'outil CIPEGE étant 
de fournir une information aux étudiants sur les filières les plus porteuses en matière d'emploi, nous espérons néanmoins, qu'à terme, cette information sera exploitée systématiquement par les nouveaux entrants afin de rendre leur décision plus rationnelle (ou du moins, moins irrationnelle).

Notre modèle logistique utilise des données administratives annuelles sur le nombre d'inscrits et de diplômés par université, filière et niveau de diplôme, des données individuelles sur les parcours d'études des étudiants recueillies dans le serveur des universités "Apogée ", ainsi que des données de l'enquête d'insertion des diplômés dans les 18 mois suivant l'obtention de leur diplôme, recueillies chaque année par le ministère de l'Enseignement supérieur et de la Recherche.

Le critère d'optimisation traduit l'objectif de commande, à savoir la poursuite de la trajectoire de référence. Le Graphique 3 nous permet d'écrire :

$$
\begin{aligned}
& y_{d}(t)=y_{\text {ref }}(t)-e(t)=y_{\text {ref }}(t)-\left(y_{p}(t)-y_{m}(t)\right)(7) \\
& y_{d}(t)-y_{m}(t)=y_{\text {ref }}(t)-y_{p}(t) .(8)
\end{aligned}
$$

Imposer à la sortie du procédé $y_{p}$ de poursuivre la trajectoire de référence $y_{r e f}$ est équivalent à imposer à la sortie du modèle $y_{m}$ de poursuivre la trajectoire désirée $y_{d}$. On suppose que l'erreur $e=y_{p-} y_{m}$, calculée à chaque nouvelle mesure, est constante sur $N_{p}$. Ce signal, représentant les perturbations et les erreurs de modélisation, constitue le retour d'information qui va impacter la trajectoire de référence $y_{\text {ref }}$. La trajectoire de référence $y_{\text {ref }}$ étant connue sur l'horizon de prédiction, la trajectoire désirée peut être calculée de la façon suivante :

$$
y_{d}(k)=y_{\text {ref }}(k)-e(t), k \in\left[t+1, t+N_{p}\right] \text {. }
$$

L'objectif de commande s'écrit comme un problème de minimisation de la fonction de coût $J$, fonction quadratique de l'erreur de poursuite $\varepsilon_{\rho}=\mathrm{y}_{\mathrm{d}}-y_{m}$ :

$$
\min _{\tilde{u}} J(u)=\sum_{k=t+1}^{t+N_{p}} \varepsilon_{p}(k)^{T} Q(k) \varepsilon_{p}(k)+\delta u(k-1)^{T} R \delta u(k-1)
$$

où $Q$ et $R$ sont des matrices symétriques définies $\quad$ positives, $\quad \delta u(k-1)=u(k-1)-u(k-2)$, $\tilde{u}=\left\{u(t), u(t+1), \ldots, u\left(t+N_{c}\right), \ldots, u\left(t+N_{p}-1\right)\right\}$ est la séquence de $\mathrm{Nc}_{c}$ commandes à déterminer avec $N_{c}<N_{p}$. Seule la première commande u(t) est simultanément appliquée au procédé et au modèle interne. La procédure est réitérée à l'instant d'échantillonnage suivant, conférant robustesse et efficience à la commande prédictive. 


\section{Simuler l'impact des stratégies institutionnelles sur l'employabilité}

Dans cet article, nous ne présentons que les procédures et résultats des simulations pour les diplômés de Master $(j=5)$. Pour les diplômés de Licence $(j=3)$, ces éléments sont disponibles sur demande auprès des auteurs.

La commande testée dans le cadre de ces simulations agit sur le nombre d'étudiants inscrits en géosciences en début de diplôme (Licence 1 et Master 1) pour répondre aux variations de la demande des marchés.

Grâce à une procédure d'identification. nous obtenons un modèle qui reproduit le procédé avec un taux d'erreur relative $\left(\mathrm{e}=y_{p}-y_{m}\right)$ de 9\% (cf. Courtial et Garrouste, 2014, pour une description détaillée de la procédure d'identification).

Le Graphique 4 révèle une trajectoire non-linéaire de l'employabilité mesurée. La forte augmentation du nombre de diplômés de Master en géosciences, en emploi entre 2008 et 2009, s'explique en partie par la réforme LMD des diplômes universitaires lancée en 2004-2005. Cette réforme consistait à harmoniser les diplômes en Europe en une structure à trois niveaux : Licence, Master, Doctorat. En France, cette nouvelle structure a progressivement remplacé les cinq niveaux de diplômes antérieurs (Diplôme d'études universitaires générales ou DEUG, Licence, Maîtrise, Diplôme d'études approfondies ou DEA et Diplôme d'études supérieures spécialisées ou DESS, Doctorat). La Maîtrise correspond aujourd'hui à un niveau Master 1 et les anciens DEA/DESS à un niveau Master 2, c'est-à-dire à un diplôme de Master. La réforme LMD a essentiellement touché les étudiants inscrits en première année à partir de 2004-2005. Parmi les étudiants de la première cohorte, nous observons une forte augmentation du nombre de diplômés au niveau Master en raison de la disparition de la Maîtrise.

Nous simulons le modèle de prédiction interne en fixant les horizons de prédiction et de commande respectivement à 5 ans et 4 ans $\left(N_{p}=5, N_{c}=4\right), R=[10 ; 0.1]$ et $Q(j)=Q(1)^{j}$ avec $Q(1)=2$, ce qui revient à pondérer de plus en plus fortement les erreurs de poursuite au fur et à mesure que le temps passe, afin de donner plus d'importance à l'objectif final. L'horizon de prédiction est fixé à cinq ans pour tenir compte du fait qu'une modification du nombre d'inscrits en première année de Licence n'aura d'impact sur le nombre de diplômés de Master qu'au bout de cinq ans, au plus tôt. 


\section{Graphique 4. Employabilité de référence mesurée et employabilité simulée au niveau Master (Bac + 5)}

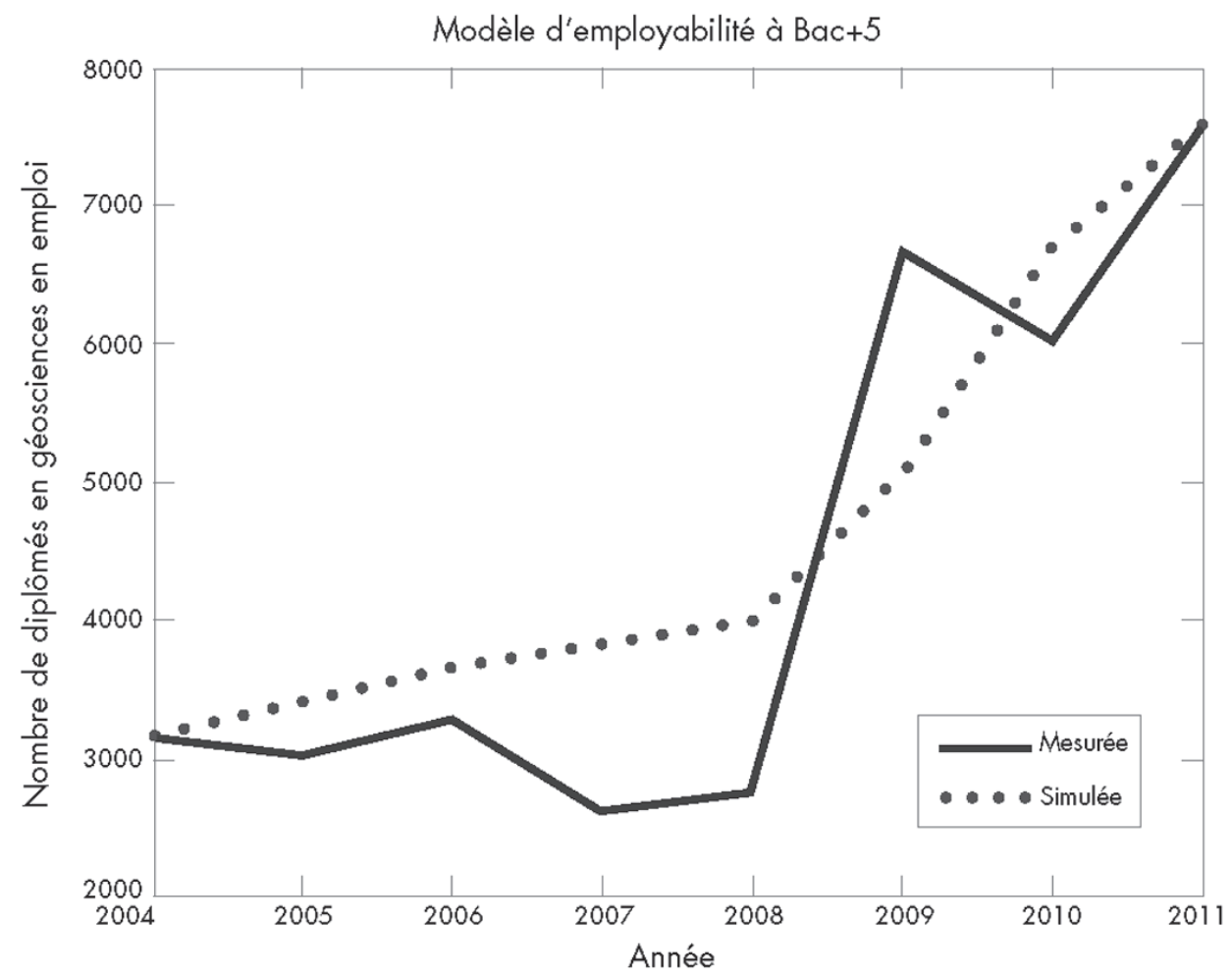

Note : Les estimations d'employabilité obtenues par l'outil CIPEGE, après avoir pris en compte les capacités de formation des universités françaises (ligne pointillée sur le graphique), sont comparées à l'employabilité de référence mesurée par notre modèle d'appariement (ligne pleine sur le graphique). L'outil de commande prédictive retenu dans cet exemple est le nombre d'inscrits en géosciences au niveau M1. L'outil CIPEGE ajuste le nombre d'inscrits au nombre de diplômés nécessaires pour converger vers la trajectoire de référence (ligne pleine sur le graphique).

Source : Estimations des auteurs de l'outil CIPEGE de simulation de l'employabilité pour les diplômés en géosciences au niveau Bac + 5 . 


\section{Graphique 5. Employabilité prédite en géosciences pour les diplômés} de Master 2012-2024

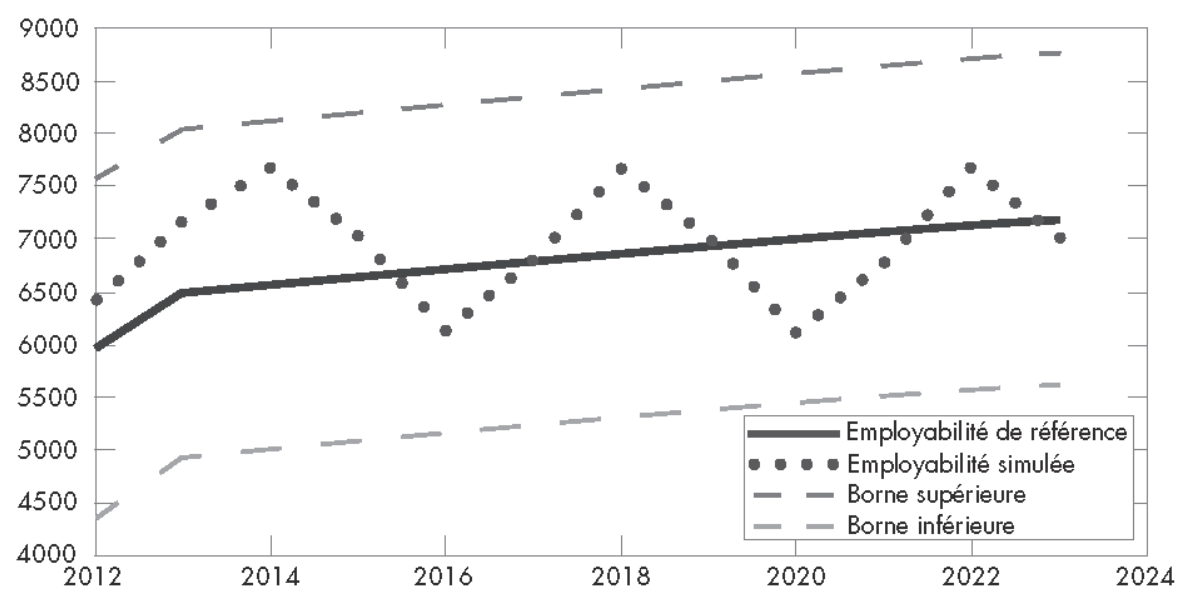

Nombre désiré d'étudiants inscrits à Bac+4

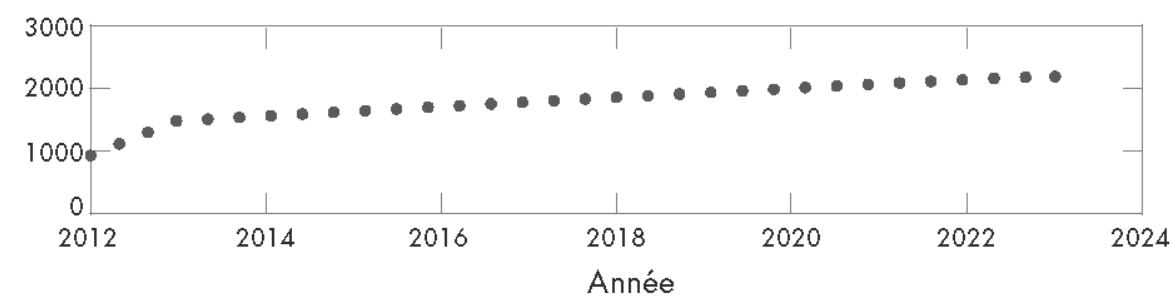

Note : Les erreurs d'estimations du modèle simulé, par rapport à la trajectoire de référence (Graphique 4), sont prises en compte par l'algorithme d'optimisation afin d'ajuster le processus à l'horizon 2025 (ligne pointillée sur le cadran haut du graphique) dans un intervalle borné correspondant à l'acceptation d'une variabilité de +/- 10\% par rapport à la valeur cible (cf. borne supérieure et inférieure, ligne hachurées). L'outil CIPEGE estime le nombre d'inscrits nécessaire en M1 afin de produire le nombre de diplômés de M2 souhaité (cadran bas du graphique) pour converger vers la trajectoire de référence (ligne pleine sur le graphique).

Source : Estimations des auteurs de l'outil CIPEGE de simulation de l'employabilité pour les diplômés en géosciences au niveau Bac +5 . 
Le Graphique 5 présente le résultat de ces simulations et montre que la sortie du modèle poursuit la trajectoire de référence tout en restant à l'intérieur de l'intervalle de confiance. À chaque instant $t$, l'algorithme d'optimisation prend en compte l'écart entre l'employabilité prédite et l'employabilité de référence et le minimise en ajustant en $t+1$, la valeur de la variable d'action (c'est-à-dire le nombre d'étudiants à inscrire afin de produire le nombre de diplômés demandé par le marché du travail). L'écart entre l'employabilité prédite converge vers l'employabilité de référence par un processus de tâtonnement. Au vu de la convergence atteinte par notre modèle (Graphique 5), ces simulations prouvent la capacité de l'outil CIPEGE à simuler l'impact de certaines actions menées au niveau universitaire (ici, agir sur le nombre d'étudiants inscrits en première année) sur l'employabilité des étudiants.

Notre analyse du rôle du flux d'entrants dans les filières de géosciences sur l'employabilité révèle que, comparativement aux inscriptions actuelles, les universités françaises devront plus que doubler le nombre d'inscrits en première année de licence d'ici 2020, afin d'apporter une réponse adéquate à la demande des marchés en termes de compétences en géosciences de niveau Master.

D'une part, le CIPEGE anticipe la demande effective d'un certain type de compétences, par secteur d'activité et type de poste, ce qui constitue une information stratégique pour les universités et les étudiants. D’autre part, cet outil peut révéler la capacité des universités à répondre à la demande effective des marchés en "produisant " une offre de compétences adéquate. Cette information est pertinente pour les secteurs fortement dépendants des ressources humaines dans leur processus de planification stratégique. L'outil CIPEGE a donc une pertinence à la fois pour les universités, les étudiants et le milieu industriel.

\section{Conclusion}

Cet article présente les résultats préliminaires d'un outil innovant de prospection de l'employabilité des diplômés en géosciences et environnement issus des universités françaises.

Pour la première fois, une approche de commande prédictive sur horizon glissant, largement utilisée en Automatique (prédiction des trajectoires des satellites ou autres robots), est appliquée à une problématique purement économique. Le choix de cette approche réside dans sa capacité à tenir compte des perturbations et autres erreurs de modélisation inhérentes à toute estimation par la structure de leur modèle de prédiction interne (MI). En complétant la correction d'erreur d'estimation appliquée dans notre modèle d'appariement entre l'offre et la demande de compétences, qui définit la trajectoire d'employabilité de référence, cette correction d'erreur de modélisation par la structure MI a pour rôle d'assurer la fiabilité et la robustesse de nos résultats. Comme le montrent nos résultats préliminaires, cet objectif semble atteint. 
Le taux d'erreur relative entre le modèle interne de prédiction et le procédé réel est inférieur à $10 \%$, ce qui est statistiquement très satisfaisant. Il révèle une capacité de l'outil CIPEGE à atteindre les objectifs d'employabilité avec une probabilité de plus de $90 \%$.

Un autre avantage de cette approche réside dans sa structure, qui permet de définir les commandes prédictives (c'est-à-dire les actions qui peuvent être mises en place par les universités afin d'atteindre les objectifs définis par la trajectoire de référence) de manière exogène.

Par exemple, la commande prédictive testée dans cet article est le nombre moyen d'inscrits en M1 nécessaire pour atteindre le nombre de diplômés en géosciences demandé par le marché du travail. D'autres commandes incluent les taux de redoublement ou de rétention, le taux de réussite, etc. Cette exogénéité permet ainsi de tester un nombre illimité de commandes sans altérer la structure du modèle. L'outil CIPEGE sera donc utilisé pour tester de nouvelles commandes, identifiées en étroite collaboration avec le ministère de l'Enseignement supérieur et de la Recherche et les Présidences universitaires.

Enfin, les courbes de tendance de l'emploi par secteur d'activité et par niveau d'occupation, que nous dérivons du modèle de l'employabilité de référence, informent sur les tendances fortes du marché de l'économie verte. Elles contribuent à alimenter le débat sur les décisions stratégiques en matière d'investissements industriels et de ressources humaines. Les résultats du CIPEGE vont ainsi être présentés et discutés régulièrement avec les industriels français afin de valider leur pertinence. Notre système de correction continue sur horizon glissant nous permettra d'intégrer ces informations au fil de l'eau. Il s'agira d'ajuster notre estimation des tendances fortes et d'identifier les signaux faibles importants.

Soulignons, néanmoins, que ce type d'outil, reposant sur un concept d'appariement, n'est pertinent que dans le cadre de formations dites « réglementées » ou pour lesquelles des objectifs métiers ont été clairement définis, telles que les géosciences, la médecine, le numérique numérisant (c'est-à-dire le codage informatique) ou la chimie. En outre, en fonction de l'ampleur des bouleversements que le verdissement de l'économie est susceptible d'apporter à la nomenclature des métiers dans les années à venir, nos résultats risqueront de perdre en justesse par manque de points d'observation. À cet égard, afin de prendre en compte cette incertitude inhérente à un secteur en pleine émergence et à des comportements individuels parfois irrationnels, nous travaillons sur une nouvelle version de l'outil CIPEGE, qui portera sur une estimation par intervalles. Cette approche vise à estimer les valeurs plausibles de l'employabilité prédite, plutôt que ses valeurs absolues, et ainsi à accroître la robustesse et la pertinence socio-économique et politique de l'outil CIPEGE. 


\section{Bibliographie}

Alessio A. \& Bemporad A. (2009), "Nonlinear Model Predictive Control", Lecture Notes in Control and Information Sciences, vol. 384.

Arjona Perez E., Garrouste C. \& Kozovska K. (2010), “Towards a benchmark on the contribution of Education and Training to Employability: a discussion note", JRC Scientific and Technical Reports EUR24147EN, Joint Research Center, Ispra.

Boaten S.K., Garrouste C., Jouhette S. (2011), "Measuring Transition from School to Workinthe EU : Role of the data source", Conference paper presented at the Conference Catch the Train: Skills, Education and Jobs, Brussels, June.

Borras I., Legay A. \& Romani C. (2008), «Les choix d'orientation à l'épreuve de l'emploi ». Notes Emploi Formation 30, Céreq, Marseille.

Camacho E.\& Bordons C. (2007), Nonlinear Model Predictive Control: An Introductory Review. Assessment and Future Directions of Nonlinear Model Predictive Control, LNCIS358, Springer-Verlag Berlin Heidelberg, Berlin, pp. 1-16.

Cambridge Econometrics (2011), Studies on Sustainability Issues: Green Jobs, Trade and Labour, Cambridge Econometrics, Cambridge, UK.

CEDEFOP (2008), Terminology of European Education and Training Policy, European Union Publications Office, Luxembourg.

Cole H. \& Rogerson R. (1996), "Can the Mortensen-Pissarides Matching Model Match the Business Cycle Facts?”, University of Minneasota Working Paper.

Couppié T., Giret J.-F., Lopez A. (2009), « Obtenir un emploi dans son domaine de formation : un enjeu très relatif dans l'insertion des jeunes ", Dossier Céreq de l'Insee-Référence Formations et emploi, pp. 43-56.

Courtial E. \& Garrouste C. (2014), Model Predictive Control Strategy to Forecast Employability in Earth Sciences, Conference paper presented at the 19th World Congress of The International Federation of Automatic Control, Cape Town, South Afric, August.

Cutuli G. \&Guetto R.(2013), "Fixed-Term Contracts, Economic Conjuncture, and Training Opportunities: A Comparative Analysis Across European Labour Markets", European Sociological Review, vol. 29(3), pp. 616-629.

Davis S.J., Haltiwanger J., Schuh S .(1996), Job Creation and Destruction, MIT Press, Cambridge, MA.

DeGrip A., van Loo J. \& Sanders J. (2004), "The Industry Employability Index: Taking Account of Supply and Demand Characteristics", International Labour Review, 143(3), pp. 211-233. 
Gazier A. (1998), Employability: Concepts and Policies, European Commission, European Employment Observatory Research Network, Bruxelles.

Harvey H., Geall V., Moon S. (1998), Work Experience: Expanding Opportunities for Undergraduates, Centre for Researchinto Quality, Birmingham.

Hillage J., Pollard E. (1998), Employability:Developing a Framework for Policy Analysis, Department for Education and Employment (DfEE), Research Report n ${ }^{\circ}$ R85.

INSEE (2012), Dossier - Définir et quantifier l'économie verte, L'économie française.

McFadden D. (1974), Conditional logit analysis of qualitative choice behavior, in Zarembka P. (ed.), Frontiers in Econometrics, Academic Press, New York, pp. 105-142.

McQuaid R., Lindsay C. (2005), "The Concept of Employability", Urban Studies, vol. 42(2), pp. 19-219.

Mortensen D.T. (1994), "The Cyclical Behavior of Job and Worker Flows", Journal of Economic Dynamics and Control, vol. 18, pp. 1121-1142.

Mortensen D. \& Pissarides C. (1994), "Job creation and job destruction in the theory of unemployment”, Review of Economic Studies, vol. 61(3), pp. 397-415.

Oliver E.A. (2012), “Living fly? How Europe's science researchers manage mobility, fixed term employment and life outside work", The International Journal of Human Resource Management, vol. 23(18), pp. 385-3871.

O’Connor J.S. (2013), “Non-Standard Employment and European Union Employment Regulation", in Max Koch et Martin Fritz (Eds.), Non-Standard Employment in Europe Paradigms, Prevalence and Policy Responses, Palgrave, Houndmills, Basingstoke, England, pp. 46-63.

Organisation Internationale du Travail (2012), Vers le développement durable : Travail décent et intégration sociale dans une économie verte, OIT, Genève.

Petrongolo B. \& Pissarides C. (2001), "Looking into the blackbox: a survey of the matching function”, Journal of Economic Literature, vol. 39 (2), pp. 390-431.

Pissarides C. \& Mortensen D. (1999), "New Developments in Models of Search in the Labour Market”, CEPR Discussion Paper, n² 2053, January.

Programme des Nations Unies pour l'Environnement (2011), Vers une Economie Verte, PNUE, France.

Qin S.J. \& Badgwell T.A. (2003), "A survey of industrial model predictive control technology”, Control Engineering Practice, vol. 11, pp. 733-764.

Wilson R.A. \& al. (2010), Skills supply and demand in Europe: medium-term forecast up to 2020, Cedefop, Thessaloniki. 